\title{
Recent Advances in Novel Topological Materials
}

\author{
Guang Bian ${ }^{1, *} \mathbb{D}$ and Tay-Rong Chang ${ }^{2}$ \\ 1 Department of Physics and Astronomy, University of Missouri, Columbia, SC 65211, USA \\ 2 Department of Physics, National Cheng Kung University, Taiwan 70101, Taiwan; \\ u32trc00@phys.ncku.edu.tw \\ * Correspondence: biang@missouri.edu
}

Received: 3 February 2020; Accepted: 5 February 2020; Published: 7 February 2020

Ever since the experimental discovery of the first 2D and 3D topological insulators, there have been intense emerging worldwide research activities in searching for and identifying new topological phases of condensed matter. In recent years, we have witnessed the laboratory-based realization of numerous novel topological materials, such as topological crystalline insulator, Weyl semimetals and new fermion matters. The interest in this topic arises from not only the realization of exotic theoretical concepts in fundamental physics, but also the promise of device applications, which can potentially revolutionize the entire Si-based electronics industry. This special issue on "Recent Advances in Novel Topological Materials" covers a broad range of the physical and chemical properties of novel topological materials including $\mathrm{Bi}$ quantum films, trigonal $\mathrm{Te}, \mathrm{Bi}_{2} \mathrm{Te}_{3}$, ferroelectric $\mathrm{GeTe}$, bismuthene and topological photonic crystals.

Bismuth has been a key element in the discovery and development of topological insulator materials. Previous theoretical studies indicated that Bi is topologically trivial and it can transform into the topological phase by alloying with $\mathrm{Sb}$. However, recent high-resolution angle-resolved photoemission spectroscopy (ARPES) measurements strongly suggested a topological band structure in pure $\mathrm{Bi}$, conflicting with the theoretical results. The puzzle was solved by Chang et al. [1] when they established experimental criteria for detecting the band topology of Bi. They found that Bi quantum films in topologically trivial and nontrivial phases respond differently. The quantum confinement effectively enlarges the energy gap in the band structure of Bi films and enables a direct visualization of the $Z_{2}$ topological invariant of $\mathrm{Bi}$.

Basnet et al. reported the synthesis by using low-pressure physical vapor deposition [2]. Raman spectra and high mobility electronic transport attest to the highly crystalline nature of the wires. A comparison of Raman spectra for both straight and curved nanowires indicates a breathing mode that is significantly broader and shifted in frequency for the curved wires. Domain-wall (DW) dark pulses from a thulium-doped fiber laser incorporating a topological insulator saturable absorber (SA) were experimentally demonstrated by Koo et al. [3]. The bulk-structured $\mathrm{Bi}_{2} \mathrm{Te}_{3}$ was used as the $\mathrm{SA}$, which was constructed on a fiber ferrule platform through the deposition of the $\mathrm{Bi}_{2} \mathrm{Te}_{3}$, mixed with distilled water. The DW dark pulses were generated from a thulium-doped fiber laser cavity with a dual wavelength at 1956 and $1958 \mathrm{~nm}$. The bulk ferroelectric structure of GeTe crystals and thin films was investigated by Kriegner et al. [4]. The experimental results show that, at room temperature, there is a preferential ferroelectric order that is remarkably consistent with theoretical predictions from ideal GeTe crystals. This robust self-poled state has high piezoelectricity and additional poling reveals persistent memory effects. Tsai et al. showed that the topological interface mode can emerge in a one-dimensional elastic string system which consists of two periodic strings with different band topologies [5]. To verify their topological features, the Zak phase of each band was calculated and reveals the condition of topological phase transition accordingly. Hu et al. studied the transformations of the topological phase and the edge modes of double-bilayer bismuthine [6]. At a critical spacing of $2 \AA$, a topological phase transition from a topological insulator to a band insulator resulting from a band 
inversion between the highest valence band and the second lowest conduction band was observed, and this was understood based on the particular orbital characters of the band inversion-involved states. Huang et al. numerically demonstrated the reconfigurable microwave photon analogues of topological insulator (TIs) in a triangular lattice of elliptical cylinders, according to the theory of topological defects [7]. Multiple topological transitions between the trivial and nontrivial photonic phases can be realized by inhomogeneously changing the ellipse orientation, without altering the lattice structure. This approach provides a new route towards freely steering light propagations in dielectric photonic crystals (PCs), which has potential applications in the areas of topological signal processing and sensing. Finally, Sun et al. proposed a photonic topological insulator (PTI) utilizing a pair of double-degenerate transverse electric (TE) and transverse magnetic (TM) polarizations to mimic the spin-up and spin-down states of the electron [8]. The unidirectional polarization-dependent transportation of TE and TM edge states can be realized in this system based on computer simulations.

The present special issue on "Recent Advances in Novel Topological Materials" can be considered as a review of the progress that has been achieved over the past several years in the synthesis and characterization of topological materials.

Conflicts of Interest: The authors declare no conflict of interest.

\section{References}

1. Chang, T.-R.; Lu, Q.; Wang, X.; Lin, H.; Miller, T.; Chiang, T.-R.; Bian, G. Band Topology of Bismuth Quantum Films. Crystals 2019, 9, 510. [CrossRef]

2. Basnet, R.; Doha, M.H.; Hironaka, T.; Pandey, K.; Davari, S.; Welch, K.M.; Churchill, H.O.H.; Hu, J. Growth and Strain Engineering of Trigonal Te for Topological Quantum Phases in Non-Symmorphic Chiral Crystals. Crystals 2019, 9, 486. [CrossRef]

3. Koo, J.; Ashok, N.; Kim, D.H.; Shin, W. $\mathrm{Bi}_{2} \mathrm{Te}_{3}$ Topological Insulator for Domain-Wall Dark Pulse Generation from Thulium-Doped Fiber Laser. Crystals 2019, 9, 337. [CrossRef]

4. Kriegner, D.; Springholz, G.; Richter, C.; Pilet, N.; Müller, E.; Capron, M.; Berger, H.; Holý, V.; Hugo Dil, J.; Krempaský, J. Ferroelectric Self-Poling in GeTe Films and Crystals. Crystals 2019, 9, 335. [CrossRef]

5. Tsai, Y.-W.; Wang, Y.-T.; Luan, P.-G.; Yen, T.-J. Topological Phase Transition in a One-Dimensional Elastic String System. Crystals 2019, 9, 313. [CrossRef]

6. Hu, H.; Shi, Z.; Wang, P.; Zhou, W.; Chiang, T.-C.; Wang, X. Transformation of the Topological Phase and the Edge Modes of Double-Bilayer Bismuthene with Inter-Bilayer Spacing. Crystals 2019, 9, 266. [CrossRef]

7. Huang, H.; Huo, S.; Chen, J. Reconfigurable Topological Phases in Two-Dimensional Dielectric Photonic Crystals. Crystals 2019, 9, 221. [CrossRef]

8. Sun, X.-C.; He, C.; Liu, X.-P.; Zou, Y.; Lu, M.-H.; Hu, X.; Chen, Y.-F. Photonic Topological States in a Two-Dimensional Gyrotropic Photonic Crystal. Crystals 2019, 9, 137. [CrossRef]

(C) 2020 by the authors. Licensee MDPI, Basel, Switzerland. This article is an open access article distributed under the terms and conditions of the Creative Commons Attribution (CC BY) license (http://creativecommons.org/licenses/by/4.0/). 